\title{
Für eine Europäische Sicherheitspolitik aus einem Guss Sieben Vorschläge für eine Grüne Sicherheitsstrategie
}

\author{
Angelika Beer*
}

\begin{abstract}
In addressing the task of building peace, an »integrated European security policy « is the most appropriate instrument. In future, all the foreign and security policy tools available - from humanitarian aid to development policy, from foreign trade policy to international finance policy, from diplomacy to disarmament, arms export, non-proliferation, sanctions and arms control policies, up to and including civilian and military crisis management - must be linked and integrated. Only by making coordinated, targeted and efficient use of the most appropriate policy tools is there any prospect of achieving the objective, namely to combat the causes of war and crises promptly and effectively.
\end{abstract}

Keywords: Europäische Sicherheits- und Verteidigungspolitik (ESVP), Frühwarnung, Rüstungskontrolle und Nichtverbreitung, ziviles und militärisches Krisenmanagement, Harmonisierung, parlamentarische Kontrolle

$\mathrm{N}$ ur sieben Jahre nachdem im Herbst 1998 die Entwicklung der Europäischen Sicherheits- und Verteidigungspolitik (ESVP) begann, gleicht die ESVP einer weithin sichtbaren Großbaustelle. Erste Gebäudeteile sind fertig gestellt und werden bereits genutzt. Arbeiter aus 24 der mittlerweile 25 Mitgliedstaaten der EU werkeln mit Unterstützung von Gastarbeitern aus manch befreundeter Nation daran, weitere Gebäude bezugsfertig zu machen. Etliche neue Rohbauten wachsen in den Himmel. In unregelmäßiger Folge werden Grundsteine für weitere Bauten gelegt. Überall sind Architekten dabei zu beobachten, wie sie immer noch neue Pläne für Erweiterungs- und Verbindungsbauten entwerfen oder Detailzeichnungen bereits geplanter Gebäude verändern. Noch erschließt sich dem unbedarften Betrachter das große Ganze, das Gebäudeensemble, nicht auf den ersten Blick. Und so mancher, der schon die ersten Modellentwürfe besichtigt hatte, fragt sich, ob er angesichts der vielen Neuund Umplanungen eigentlich noch einen Gesamtüberblick hat.

Trotz allem: Die ESVP wird Schritt für Schritt zu einem immer wichtigeren Bezugsrahmen für die Sicherheitspolitik der Mitgliedstaaten der EU. Mittlerweile kann die EU bereits auf zwei kleinere, abgeschlossene militärische Friedensmissionen im Kongo und in Mazedonien und auf die laufende erste größere, »Althea « in Bosnien-Herzegowina, schauen. Die EU führt zwei Polizeimissionen, eine Rechtshilfemission und eine Beobachtermission durch. Eine weitere Polizeimission ist in Planung. Sie verfügt über einen Militärausschuss, einen Militärstab, eine Verteidigungsagentur und eine Solidaritätsklausel für den Fall terroristischer Angriffe. Sie hat sich gemeinsame Planungsziele für den Aufbau ziviler und militäri-

\footnotetext{
Angelika Beer, MdEP (Fraktion DIE GRÜNEN/EFA), ist Mitglied des Auswärtigen Ausschusses und des Unterausschusses für Sicherheit und Verteidigung des Europäischen Parlaments. Dieser Beitrag bezieht sich auf eine umfassendere Broschüre mit demselben Titel (unter http:// www.angelika-beer), erhältlich über karsten.vollrath@gruene-europa.de.
}

scher Krisenmanagementkapazitäten gesetzt, deren erste Teile bereits umgesetzt wurden und die um anspruchsvollere erweitert wurden. Die EU hat eine Sicherheitsstrategie entworfen und entwickelt in etlichen Bereichen, so zum Beispiel bei der Proliferationsverhinderung und bei der Terrorismusbekämpfung, gemeinsame außen- und sicherheitspolitische Politiken.

Europa ist ein weltpolitischer Akteur. Europa muss sich dieser Verantwortung stellen. Der ESVP-Prozess verdeutlicht, dass Sicherheitspolitik künftig in wachsendem Maße nicht mehr national, sondern in multinationaler Kooperation oder gar supranational gestaltet werden wird. Die EU wird künftig »aus einer Hand « eine Vielzahl außen- und sicherheitspolitischer Wirkungsinstrumente einsetzen können, mit denen sie Konfliktursachen bekämpfen, gewaltsamen Konflikten vorbeugen und gewalttätige Konflikte einhegen, eindämmen, beenden und viele Konflikte vielleicht auch ohne Gewalt lösen kann. Es gilt sie wirksam, effizient und im Rahmen einer klugen Friedens- und Sicherheitspolitik einzusetzen, einer Politik, die nicht den Krieg, sondern den Frieden vorbereitet.

Voraussetzung dafür ist, dass die EU Friedenspolitik als ressortübergreifende Querschnittsaufgabe betrachtet. Das setzt voraus, dass Europa sich zu einem globalen Akteur entwickelt, der die Gestaltung von Weltordnung kooperativ und mit dem Ziel der Gestaltung von Friedensordnungen betreibt. Im Kern lautet die Aufgabe, Sicherheit neu und erweitert zu definieren und neue Strategien zu entwerfen, mit denen Sicherheit und Frieden gewährleistet werden können. Für die Aufgabe, Friedensordnungen zu gestalten, ist eine »Europäische Sicherheitspolitik aus einem Guss« am besten geeignet. Zu einer solchen Politik müssen in der Zukunft alle Gestaltungsmittel der Außen- und Sicherheitspolitik, von der humanitären Hilfe über die Entwicklungspolitik, die Außenwirtschafts- bis zur internationalen Finanzpolitik, von der 
Diplomatie über Abrüstung und Rüstungskontrolle, den Rüstungsexport, die Nichtverbreitungspolitik, Sanktionspolitiken und Rüstungskontrollpolitik bis hin zu den Mitteln des zivilen und militärischen Krisenmanagements verzahnt und integriert werden. Nur ein koordinierter, zielgerichteter und effizienter Einsatz der jeweils am besten geeigneten Gestaltungsmittel verspricht Erfolg, wenn es darum geht, die Krieg und Krisen fördernden Faktoren gleichzeitig und wirksam zu bekämpfen. Das heißt aber auch: Sicherheit kann im 21. Jahrhundert weder national gewährleistet noch länger vorrangig mit militärischen Mitteln geschaffen werden. Sicherheitspolitik setzt einen erweiterten Sicherheitsbegriff voraus, einen Sicherheitsbegriff, der alle Dimensionen menschlicher Sicherheit umfasst.

Das wohl größte Problem der Europäischen Union und ihrer Mitgliedstaaten auf diesem Wege besteht darin, dass die weitere Integration unter dem Vorzeichen einer weitgehend intergouvernemental organisierten sicherheitspolitischen Zusammenarbeit nur langsam, unvollständig und mit gewissen Reibungsverlusten gelingen kann. Trotzdem ist aber davon auszugehen, dass diese Form der Zusammenarbeit in der nahen Zukunft die dominante bleiben wird. Die Harmonisierung hat vorläufig Vorrang vor der Vergemeinschaftung. Auch mit ihr können weitere Fortschritte erzielt werden. Gerade deshalb aber ist es besonders wichtig, bei der weiteren Ausgestaltung der ESVP die langfristige Perspektive, das in den Europäischen Verträgen und im Verfassungsvertrag benannte Ziel einer gemeinsamen Europäischen Verteidigungspolitik und eine vergemeinschaftete Außen- und Sicherheitspolitik, nicht aus den Augen zu verlieren. Nationalen Blockaden, wie derzeit beim China-Waffenembargo oder bei der Wehrpflicht, muss daher dringend entgegengewirkt werden. Stattdessen müssen die Weichen für eine weitere Harmonisierung gestellt werden.

\section{Frühwarnung als Voraussetzung für Prävention}

Ein frühzeitiges, proaktives Krisenmanagement kann nur erfolgreich sein, wenn potentielle Krisen früh- und rechtzeitig identifiziert werden und damit nichtmilitärische Krisenmanagementkapazitäten ihre Wirksamkeit entfalten können. Eine solche Politik impliziert die Bereitschaft zum politischen »Agenda-Setting « - auch auf globaler Ebene und in den internationalen Institutionen.

Wenn in der Europäischen Sicherheitspolitik der Zukunft die Krisen- und Gewaltprävention Vorrang vor der Konflikt- und Gewalteindämmung haben soll, so erfordert dies exzellente Fähigkeiten zu einer Konfliktfrühwarnung. Denn nur die rechtzeitige Kenntnis potentiell drohender gewaltförmiger Konflikte und ihrer Ursachenstruktur ermöglichen rechtzeitige und effiziente »zivile Interventionen $«$.

\subsection{Quellen besser nutzen}

Eine gute Konfliktfrühwarnung kann aus vielen Quellen gespeist werden. Sie kann nur erfolgreich sein, wenn sie die Rahmenbedingungen schafft, damit diese Quellen auch spru- deln können. Dafür ist ein weitgehendes Umdenken erforderlich. Es setzt auf staatlicher Seite den politischen Willen voraus, an einer strategischen Neuausrichtung der Informationsgewinnung und -analyse zu arbeiten. Dabei müssen vor allem drei Grenzen überschritten werden. Es gilt

Erstens in bisher nicht gekanntem Maße Informationen über die Grenzen zwischen Zivilgesellschaft und Staat auszutauschen.

Zweitens Informationen über die Grenzen zwischen den Staaten Europas auszutauschen.

Drittens zur Stärkung eines effizienten Multilateralismus beizutragen, indem zusätzlich auch Informationen mit den Vereinten Nationen, ihren Regionalorganisationen und anderen multilateralen Institutionen ausgetauscht werden.

Dies mag zunächst sehr anspruchsvoll erscheinen, da alle drei Grenzen traditionell von Seiten des Nationalstaates und seiner Bürokratien möglichst undurchlässig gestaltet werden. Es ist aber eine zwingende Voraussetzung für eine substantiell verbesserte und effiziente Konfliktfrühwarnung, die ihrerseits wiederum erst die Möglichkeit zu einer effizienten Konfliktprävention eröffnet.

Hier sei nur ein Beispiel stellvertretend für andere ausgeführt: Wissenschaftler, Mitarbeiter von örtlich tätigen Nichtregierungsorganisationen, Entwicklungshelfer oder auch Journalisten und örtliche Mitarbeiter europäischer Firmen - all diese Akteure generieren nutzbares Wissen, das viel besser und intensiver für die Konfliktfrühwarnung genutzt werden kann, als dies heute geschieht. Das gleiche gilt auch für das Wissen von diplomatischen Vertretungen oder Mitarbeitern aus der staatlichen und privaten Entwicklungszusammenarbeit. Die EU und ihre Mitgliedstaaten müssen Wege entwickeln, dieses umfängliche und oft sehr aktuelle Wissen auf der analytischen Ebene zu integrieren und auszutauschen. Dann eröffnen sie sich Möglichkeiten, deutlich präzisere Lagebilder und -einschätzungen zu entwickeln, die für die Konfliktprävention genutzt werden können. Voraussetzung dafür aber ist, dass die Grenzen zwischen dem Wissen staatlicher Stellen und dem zivilgesellschaftlichen Wissen durchlässig gemacht werden. Nur dann ist mit einer ausreichend breiten Beteiligung aus der Zivilgesellschaft zu rechnen.

\subsection{Frühwarnung neu organisieren}

Der bereits vereinbarte Austausch der für die Konfliktprävention relevanten Informationen zwischen der EU und den Vereinten Nationen, ihren Unter- und Regionalorganisationen sowie anderer geeigneter Partner auf Initiative der EU in einem gemeinsamen Projekt muss weiter systematisiert werden, und es müssen geeignete Wege gesucht werden, wie diese Informationen öffentlich für alle Beteiligten bereitgestellt werden können. Die Vereinten Nationen haben bereits erste Informationsbörsen dieser Art geschaffen. Die Öffentlichkeit der Informationen ist geboten, um eine breite Beteiligung aus der Zivilgesellschaft zu ermöglichen. Sie hat zudem den Charme, die Beteiligung der Zivilgesellschaften aus Ländern zu ermöglichen, die selbst an einem solchen Informationssystem nicht teilnehmen wollen. 


\section{Für eine umfassende Politik der Nichtweiter- verbreitung, Rüstungskontrolle und Abrüstung}

Als konsequente Umsetzung der Europäischen Sicherheitsstrategie (ESS) müssen Rüstungskontrolle, Nichtweiterverbreitung und Abrüstung als wesentliche Instrumente der Konfliktprävention verstanden werden. Europas Strategie zur Bekämpfung der Proliferation von Massenvernichtungswaffen, aber auch zahlreiche rüstungskontrollpolitische Initiativen der EU in vielen Bereichen vom Kleinwaffenhandel bis zur Verhinderung der Proliferation von Massenvernichtungswaffen belegen, dass diese Aufgabe seitens der EU im Grundsatz erkannt und angenommen wurde. Die Europäische Union muss ihr Potential nutzen, um Rüstungskontrolle und Nichtverbreitung in dieser Funktionalität weiter zu stärken.

\subsection{Rüstungskontrolle, Abrüstung und Nicht- weiterverbreitung vor Neuordnung}

Die Politikbereiche der Rüstungskontrolle und der Nichtverbreitung stehen vor einem substantiellen Umbruch. Beide wurden als Instrumente zwischenstaatlicher, rechtlicher Verregelung geschaffen. Dies schränkt ihre Wirkmöglichkeit gegenüber nichtstaatlichen Akteuren ein, denen aber ihrerseits eine wachsende Bedeutung in der Sicherheitspolitik zukommt.

Die nukleare Bedrohung ist nicht gebannt. Die diesjährige Überprüfungskonferenz des Atomwaffensperrvertrages (NPT) ist gescheitert. Umso wichtiger sind neue multilaterale Ansätze zum Durchbrechen der drohenden nuklearen Rüstungsspirale. Alle De-facto-Atommächte müssen das Abkommen unterzeichnen, das Zusatzprotokoll zur Überprüfung der Einhaltung muss für alle Unterzeichner verbindlich werden und auch die zivile Nutzung von Nukleartechnik muss eingeschränkt werden.

Als erster und wegweisender Schritt zur nuklearen Abrüstung muss deswegen der Abzug der in Europa stationierten 480 US-Atomsprengköpfe erfolgen und damit die nukleare Teilhabe beendet werden. Darüber hinaus gilt es zu verhindern, dass die USA neue Atomwaffen entwickeln. Die Entwicklung der so genannten »Mini-Nukes« würde gegen das Nichtverbreitungsregime verstoßen und alle Abrüstungsbemühungen konterkarieren.

\subsection{Der Europäische Ansatz im Atomstreit mit dem Iran}

Das intensivierte Engagement der EU zur Stärkung der Nichtweiterverbreitung kommt auch im Atomstreit mit dem Iran zum Ausdruck: Die »EU-3« Deutschland, Frankreich und Großbritannien versuchen den Iran dazu zu bewegen, seine Pläne für einen nuklearen Brennstoffkreislauf endgültig aufzugeben und insbesondere alle Aktivitäten, die im Zusammenhang mit der Urananreicherung stehen, verifizierbar einzustellen. Da der Iran zu einem solchen unilateralen Verzicht rechtlich nicht verpflichtet ist, werden ihm parallel Kompensationen für einen Verzicht offeriert.
Dieser schwierige Verhandlungsprozess zwischen EU und Iran macht die grundsätzlichen Unterschiede von ESS und NSS deutlich: Die Europäische Sicherheitsstrategie verfolgt eine friedliche Strategie zur Verhinderung der Proliferation. Die Nationale Sicherheitsstrategie der Bush-Administration setzt letztlich auf Präventivschläge. Um ein Desaster im Mittleren und Nahen Osten zu verhindern, gibt es zu den Verhandlungen der EU keine Alternative.

\subsection{Die Notwendigkeit eines strategischen Gesamtkonzeptes}

Die begonnene, sinnvolle Arbeit muss weitergeführt werden. Ihre Einzelelemente sollten in ein strategisches Gesamtkonzept überführt werden, dessen Aufgabe es zugleich ist, zu einer weltweiten Neu- und Wiederbelebung rüstungskontrollpolitischer und abrüstungspolitischer Verhandlungen zu kommen. Rüstungskontrolle und Nichtverbreitung können auch dann praktiziert werden, wenn sich einzelne wichtige Staaten zunächst noch nicht beteiligen. Dies zeigen so unterschiedliche Beispiele wie der NPT und der Ottawa-Vertrag.

Die EU muss in den kommenden Jahren ein strategisches Gesamtkonzept zur Abrüstung und Nichtweiterverbreitung entwickeln, das diesem Ziel dient und von der EU international befördert wird. In ein solches Konzept könnten verschiedene Elemente eingehen. Dazu gehören insbesondere eine Verbreiterung bzw. Universalisierung der Gültigkeit bestehender Abkommen, ein integriertes Konzept technischer und finanzieller Abrüstungs- und Nichtverbreitungshilfen sowie Vorschläge für neue Vereinbarungen etwa im Bereich der Weltraumbewaffnung und der kleinen und leichten Waffen.

\subsection{Nichtstaatliche Akteure einbinden}

Angesichts der zunehmenden Bedeutung von nichtstaatlichen Akteuren in Sicherheitsfragen bedarf es dringend einer Konzeption für ein effizientes Einwirken auf diese Akteure mittels Rüstungskontrolle und Nichtverbreitung. Solange eine solche Konzeption noch nicht vorliegt, können die rüstungskontrollpolitischen und Nichtverbreitungsziele europäischer Politik auch durch eine gezielte Kooperation mit Nichtregierungsorganisationen gefördert werden. Ein Beispiel stellt »Geneva Call« dar, ein Zusammenschluss, in dem sich nichtstaatliche Gewaltakteure dazu verpflichtet haben, das Ottawa-Abkommen über Landminen einzuhalten, diese Einhaltung durch einen Verifikationsmechanismus überprüfen zu lassen und ggf. bei Verletzungen auch Sanktionen hinzunehmen. Über eine Erweiterung von »Geneva Call« könnte z.B. auch eine verifizierbare Einhaltung der Regeln des humanitären Kriegsvölkerrechtes durch erste nichtstaatliche Akteure initiiert werden.

\section{Sicherheitspolitik weiter denken - am Beispiel Energiepolitik}

Beiträge zur Sicherheitspolitik, vor allem zur Konfliktprävention, müssen künftig auch aus Politikfeldern kommen, die bislang nicht unter dem Gesichtspunkt ihrer sicherheits- 
politischen Implikationen diskutiert wurden. Das zeigt das Beispiel der Energiepolitik. Zwar wird oft über potentielle Gefährdungen unserer Energieversorgung diskutiert manchmal fließen auch Argumente über die Notwendigkeit, Fragwürdigkeit oder die Kosten einer notfalls militärischen Absicherung der Energieversorgung in die Debatte ein. Äußerst selten aber wird das folgende, gewichtige Argument angeführt: Innovativ ausgerichtete Investitionen in eine neue Energie- und Energiesicherheitspolitik können Investitionen in die Sicherheit Europas sein, weil sie die Abhängigkeit Europas von Öl- und Gasimporten verringern. Sie können wesentlich zur Konfliktprävention beitragen, weil sie die Konkurrenz um die knapper werdenden fossilen Rohstoffe reduzieren, und sogar effizienter sein, als es vergleichbare Investitionen in neue militärische Fähigkeiten wären.

\subsection{Weg von Öl und Gas}

Schon heute ist die gesicherte Zufuhr von Energieressourcen nach Europa lebenswichtig. Erdöl und Gas kommen dabei eine immer größere Bedeutung zu. Beide werden überwiegend aus potentiellen Krisenregionen importiert. Wirkliche Alternativen gibt es nicht. Unkalkulierbare Preisentwicklungen, Unterbrechungen des Nachschubs oder gar längere Lieferausfälle sind nur einige der Risiken. Lange Transportwege und Transportsysteme, die gute Ziele für asymmetrische Drohungen (Piraterie, Terrorismus zur See etc.) darstellen, verschärfen die Problematik. Angesichts des wachsenden Weltenergiebedarfs wird die Nachfrage nach den endlichen Ressourcen Öl und Gas weiter steigen und sich damit die Konflikte um eine gesicherte Versorgung verschärfen.

Angesichts dieser Situation ist die Verringerung der Abhängigkeit von Öl und Gas durch eine gezielte Förderung regenerativer Energien und dezentraler Energieversorgungssysteme eine äußerst bedeutsame Investition in die Sicherheit der europäischen Staaten und ihrer Gesellschaften. Die Idee einer konzertierten Arbeit für eine energie- und sicherheitspolitische »Agenda for Change « bietet eine exzellente Möglichkeit, die Tragweite und den Charakter einer »Europäischen Sicherheitspolitik aus einem Guss « an diesem Beispiel $\mathrm{zu}$ thematisieren und zu verdeutlichen. Als Ergebnis wäre eine Europäische Energiesicherheitsstrategie, verkoppelt mit konkreten Aktionsplänen, denkbar, mit der die Europäische Union eine Konflikten vorbeugende und der eigenen Sicherheit dienliche neue Energiepolitik festschreibt.

\section{Das zivile Krisenmanagement stärken}

Das zivile Krisenmanagement der EU ist und bleibt das Standbein der Europäischen Außen- und Sicherheitspolitik. Dies wird nicht zuletzt in der ESS immer wieder deutlich. Das zivile Konfliktmanagement kann zu allen Dimensionen der Schaffung von Friedensordnungen beitragen und bei guter Planung sicherstellen, dass die Prozesse des Herstellens von Sicherheit und Stabilität, der Sicherung von Rechten und der Ermöglichung nachhaltiger wirtschaftlicher Entwicklung angemessen und ausgewogen Berücksichtigung finden. Es leistet die meisten wesentlichen Beiträge, wenn es gilt, Konfliktursachen zu begegnen, Krisen vorzubeugen und Friedensordnungen aufzubauen. Zivile Krisenmanagementfähigkeiten werden fast immer gefordert sein, wenn militärische Mittel zum Einsatz kommen. Militärische Mittel dagegen können die zivilen in manchen Fällen unterstützen und ergänzen, sie können diese aber weder ersetzen, noch ist ihr Einsatz jedes Mal vonnöten. Deshalb kommt der Weiterentwicklung und dem Ausbau der zivilen Krisenmanagementinstrumente der Union überragende Bedeutung zu. Dem zivilen Krisenmanagement der Europäischen Union stellt sich künftig ein äußerst breites Feld von Aufgaben: von der Sicherstellung der Versorgung der Bevölkerung (humanitäre Nothilfe), über den Wiederaufbau von Infrastrukturen sowie nachhaltiger wirtschaftlicher Entwicklungsmöglichkeiten und die Aufrechterhaltung öffentlicher Ordnung bis hin zur Wiederherstellung einer Rechtsordnung und zum (Wieder-)Aufbau staatlicher Institutionen und demokratischer Organe. Hinzu kommt das weite Feld der Konfliktprävention und der Ursachenbekämpfung.

\subsection{Das Stabilitätsinstrument}

Die EU verfügt bereits sowohl über ein Set an Maßnahmen aus langfristigen Länderprogrammen, als auch über Instrumente wie Entwicklungszusammenarbeit, Außenhandel, Menschenrechts- und Umweltpolitik sowie politischer Dialog und humanitäre Hilfe. Sie können jedoch nicht alle Krisen- und Konfliktsituationen abdecken. Bisher fehlt ein Instrument, welches für Situationen zur Verfügung steht, in denen mehr geleistet werden muss als kurzfristige humanitäre Hilfe und langfristige Entwicklungszusammenarbeit. Mit dem von der Kommission im September 2004 vorgeschlagenen »Stabilitätsinstrument « soll dieser Problematik begegnet werden. Auch wenn der Vorschlag insgesamt noch vage und unzureichend im Hinblick auf die Rechte des Parlamentes und die Mittelausstattung ist, sind die Ziele des neuen Instrumentes zu begrüßen. Das Stabilitätsinstrument möchte Krisenbewältigung in Drittländern solange leisten, bis diese in die »normalen « Kooperationsprogramme übergeben werden können und zudem die Zusammenarbeit bei der Bedrohung der Sicherheit auf den Gebieten Justiz, Verkehr, Demokratie und Gesundheit oder durch Massenvernichtungswaffen gewährleisten. Gleichzeitig will es den ganzen Bogen von Ratifizierung, Durchführung und Überwachung internationaler Übereinkünfte zur Frühwarnung abdecken.

\subsection{Zivile Interventionsfähigkeit herstellen}

Wollen die EU und ihre Mitgliedstaaten Friedensmissionen mit dem Ziel durchführen, zu allen Dimensionen eines Friedensprozesses und damit zur Schaffung von Friedensordnungen beizutragen, dann müssen sie auch für das zivile Krisenmanagement substantielle, gut ausgebildete, präsente und rasch verfügbare also »stehende« Kapazitäten bereithalten. Die Zusammenarbeit zwischen diesen staatlich bereitgestellten zivilen Krisenmanagement-Strukturen und den militärischen Anteilen von Friedensmissionen sollte im Voraus 
im Rahmen der EU geplant und geübt sein, damit sie reibungslos und effizient funktioniert.

$\mathrm{Zu}$ den wesentlichen weiteren Schritten gehören:

Die Schaffung eines aus Angestellten der EU und ihrer Mitgliedstaaten bestehenden Zivilen Europäischen Friedenskorps, also einer einheitlichen, dauerhaften Struktur, die Aufbau, Bereithaltung, Ausbildung und Einsatz integrierter ziviler Krisenmanagementkräfte mit vielen unterschiedlichen Fähigkeiten planen und koordinieren sowie auf bereitstehende Personalressourcen zurückgreifen kann.

Ergänzend ist ein Europäisches Freiwilligenkorps sinnvoll, dessen Personal sich aus der Zivilgesellschaft und weiteren Staatsangestellten rekrutiert. Mit dem Freiwilligenkorps können die staatlichen Personalressourcen einsatz- und aufgabenbezogen ergänzt werden.

Sinnvoll ist die Schaffung einer Europäischen Agentur für Zivile Krisenmanagementfähigkeiten, die planerische und koordinatorische Aufgaben im Blick auf die technische Ausstattung und die Infrastruktur der zivilen Krisenmanagementkräfte der EU übernimmt sowie Forschung, Entwicklung und Beschaffung koordiniert und harmonisiert.

\section{Das militärische Krisenmanagement harmoni- sieren}

Europas entstehende militärische Krisenmanagementfähigkeiten ergänzen das zivile außen- und sicherheitspolitische Instrumentarium der EU. Sie erweitern deren Handlungsmöglichkeiten, dürfen diese aber nicht dominieren.

\subsection{Ein Weißbuch für Europa}

Die EU wird auch künftig über Umfang, Auftrag und Ausrichtung ihrer militärischen Mittel entscheiden müssen. Gleiches gilt für die Kriterien, mittels derer sie und ihre Mitglieder festlegen, wann sie bereit sind, Streitkräfte einzusetzen und wann nicht. Vor diesem Hintergrund muss die Idee eines Weißbuches zur ESVP umgesetzt werden. Hierin müssen Szenarien diskutiert werden, Ziele und Herausforderungen sowie Einsatzvoraussetzungen bestimmt werden.

Kennzeichnend für das Spektrum wahrscheinlicher, militärischer Einsätze ist es, dass zielbedingt sehr unterschiedliche Fähigkeiten gefordert und gefragt sein werden: Zum einen sind dies zur Deeskalation beitragende, die Lage stabilisierende militärische Fähigkeiten (quasi gehobener polizeilicher Natur), zum anderen unter allen Bedingungen durchsetzungsfähige, überlegene und eskalationsfähige Wirkungskräfte. Die Planung der EU, sowohl Europäische Gefechtsverbände (EBGs) als auch Europäische Eingreifverbände (ERRF) aufzustellen, entspricht im Grundsatz dieser Aufteilung.

Die Fähigkeiten Europas zur Stabilisierung werden häufiger und vor allem für längere Dauer gefordert sein, die Fähigkeiten zur Intervention etwas seltener, in kleinerem Umfang und für kürzere Dauer. Für die Mitglieder der EU und vor allem im Rahmen der EU besteht also ein vorhersehbar deutlich größerer Bedarf an Stabilisierungskräften als an
Wirkungskräften. Absehbar ist auch, dass zusätzlich Unterstützungskräfte verfügbar sein müssen, die Operationen jeder Art durch Aufklärung, Führung oder Logistik auch über große Entfernungen unterstützen können.

\subsection{Militärische Fähigkeiten verbessern}

Aus diesen Rahmenbedingungen ergeben sich erste Schlussfolgerungen:

Der bisherige Umfang der militärischen Krisenmanagementkräfte der EU ist angemessen, deren quantitativer Schwerpunkt bei den Stabilisierungskräften ist richtig gewählt und auch der Ansatz, Krisenmanagementoperationen vorrangig als integrierte zivil-militärische Operationen zu denken, ist zu begrüßen.

Das Hauptaugenmerk der Zukunft sollte vor allem auf einer Verbesserung qualitativer Merkmale liegen. Dabei kann davon ausgegangen werden, dass die Bemühungen im Rahmen des European-Capability-Action-Plan-Prozesses die Schwachstellen sowohl der ERRF als auch der EBGs weitgehend korrekt identifiziert haben bzw. werden und deshalb die fähigkeitsbezogenen Streitkräfteziele dem Bedarf entsprechen. Zweifel bestehen allerdings im Blick auf die Frage, ob Fähigkeitslücken immer mit geeigneten und effizienten Mitteln geschlossen werden. Hier seien nur die Beschaffungsentscheidungen über den A400M oder MEADS genannt.

\subsection{Personal, Einsatzkonzepte und Ausbildung als zentrale Herausforderungen}

Weitere wesentliche qualitative Verbesserungen des militärischen Krisenmanagements der EU sind nicht primär im Bereich von Bewaffnung und Ausstattung zu suchen, sondern in den Bereichen Personal, Einsatzkonzepte und Ausbildung. Hier werden die EU-Staaten nicht umhinkommen, jenseits ihrer Bemühungen um eine gemeinsame Kultur des Krisenmanagements, die mit einem EU-Verteidigungskolleg gefördert werden soll, den folgenden Aspekten besondere Aufmerksamkeit zu widmen:

Das Personal für militärische Krisenmanagementaufgaben muss in allen EU-Staaten so ausgewählt und ausgebildet werden, dass es das Ziel der Politik der EU, Friedensordnungen zu gestalten, auch qualifiziert umsetzen kann. Gemeinsame auf dieses Ziel ausgerichtete Ausbildungsvorschriften und Ausbildungsmaßnahmen wären deshalb von Vorteil.

Das Konzept der Inneren Führung und des Staatsbügers in Uniform haben sich in der Bundeswehr bewährt. Vor dem Hintergrund des militärischen Krisenmanagements gewinnen diese Konzepte neue Bedeutung und müssen an die neuen Herausforderungen angepasst werden, wenngleich sie in einem europäischen »best practice« Wettbewerb bestehen und weiterentwickelt werden müssen.

Im Bereich der zivil-militärischen Einsatzplanung und -vorbereitung gilt es, die Eigenständigkeit der zivilen Akteure insbesondere von Entwicklungsorganisationen zu respektieren und ihre gewachsenen Kontakte und die langjährigen Erfahrungen vor Ort zu berücksichtigen. 


\subsection{Den Rüstungsmarkt rationalisieren}

Wer den Ausbau der Europäischen Sicherheits- und Verteidigungspolitik (ESVP) einschließlich ihrer militärischen Komponente bejaht, muss auch die Herausbildung europäischer rüstungswirtschaftlicher Strukturen befürworten. Dies ist nicht zuletzt eine Frage der Harmonisierung, der Interoperabilität und der Ressourcenersparnis.

Die Herausforderung besteht darin, eine integrierte und abgestimmte Europäische Rüstungspolitik zu formulieren. Bei der Entwicklung und dem Aufbau entsprechender Strukturen sollte vor allem auf folgende Aspekte geachtet werden:

Eine Europäische Rüstungspolitik und die entsprechenden Instrumente müssen in funktionsfähige Prozesse der demokratischen Kontrolle eingebunden werden und so transparent wie möglich gestaltet werden.

Rüstungsindustrielle Verbände sollten nicht, wie im Kontext der WEU einen privilegierten Zugang zu den entsprechenden Planungsausschüssen erhalten und - noch vor den demokratisch gewählten Abgeordneten - zu offiziellen Partnern der Verteidigungsagentur und/oder der Kommission in Rüstungsfragen werden.

\section{Sicherheitspolitische Entscheidungen besser koordinieren}

Ein Manko europäischer Sicherheitspolitik besteht in der fehlenden Koordination sicherheitspolitischer Entscheidungen. Eine »Europäische Sicherheitspolitik aus einem Guss « kann nur implementiert werden, wenn Entscheidungen ressortübergreifend vorbereitet und getroffen werden. Dies gilt sowohl für die nationale, als auch für die intergouvernementale und die supranationale Ebene. Zugleich gilt es, die Entscheidungsvorbereitung und -findung dieser drei Ebenen zu koordinieren und - soweit es geht - zu harmonisieren.

\subsection{Der »Rat für Erweiterte Sicherheit«}

Auf nationaler Ebene wird in jedem Mitgliedstaat der EU eine exekutive sicherheitspolitische Struktur benötigt, die das Zusammenwirken aller Ressorts mit sicherheitspolitisch relevanten Instrumenten befördert, ermöglicht, koordiniert und entlang getroffener Entscheidungen sicherstellt. Um dem Kind einen Namen zu geben sei von einem »Rat für Erweiterte Sicherheit « in jedem EU-Mitgliedsland die Rede. Seine Funktion besteht in Beiträgen zur Entscheidungsvorbereitung, der Entscheidungsumsetzung, der Ressourcenzuweisung und der Vertretung der nationalen Positionen im europäischen Kontext. Wünschenswert wäre dabei insbesondere eine starke Einbeziehung zivilgesellschaftlicher Akteure.

Auf europäischer Ebene gilt es, die Entscheidungsvorbereitung, Entscheidungsfindung und Entscheidungsumsetzung hinsichtlich der sicherheitspolitischen Instrumente der Kommission besser $\mathrm{zu}$ verzahnen und $\mathrm{zu}$ integrieren. Dies dürfte durch ein Koordinationsgremium mit Fachleuten aus allen relevanten Generaldirektionen zu leisten sein, dem die Aufgabe zukäme, direktionsübergreifend Entscheidungsvor- lagen zu erarbeiten. Für den Rat stellt sich im Bereich der intergouvernementalen Zusammenarbeit eine vergleichbare Aufgabe. Hier bietet sich ein Koordinationsgremium an, das u.a. aus Vertretern der nationalen »Räte für erweiterte Sicherheit « besteht.

\subsection{Das »Gemeinsame Gremium für Erweiterte Sicherheit«}

Nicht zuletzt bedarf es aber auch der Harmonisierung, Koordination und Integration der Positionen und Ressourcen von Nationen, Rat, und Kommission, bevor Entscheidungen fallen. Auch hier empfiehlt sich - angesichts der derzeit gegebenen rechtlichen Rahmenbedingungen - die Form eines Koordinationsgremiums, das Entscheidungen vorbereitet. Dieses sollte als »Gemeinsames Gremium für Erweiterte Sicherheit« (Arbeitstitel) die Mitglieder der Koordinationsgremien des Rates und der Kommission zusammenbringen und gemeinsame Empfehlungen für die Entscheidungen von Rat und Kommission aussprechen.

\section{Transparenz und parlamentarischen Kontrolle stärken}

Eine »Europäische Sicherheitspolitik aus einem Guss « bedarf einer deutlich verbesserten parlamentarischen Kontrolle und einer höheren Transparenz für die Öffentlichkeit. Je mehr und je schneller die ESVP Fortschritte macht, desto stärker wird in der europäischen Öffentlichkeit der Wunsch nach Transparenz, nachvollziehbarer parlamentarischer Kontrolle und Überprüfbarkeit der Kosten der ESVP.

\subsection{Mitentscheidung des Europäischen Parlaments}

Parlamentarische Kontrolle und Transparenz müssen um substantielle Mitentscheidung erweitert werden. Mitentscheidung ist möglich, wenn das Parlament Mitspracherechte bei der Finanzierung der ESVP bekommt. Bei der jährlichen Haushaltsplanung sowie der darauf folgenden Haushaltskontrolle muss die Zustimmung des Parlamentes eingeholt werden. Grundvoraussetzung hierfür ist eine Debatte über die gemeinschaftliche Finanzierung von ESVP-Teilaspekten. Die Diskussion um den ATHENA-Mechanismus während der niederländischen Präsidentschaft muss weitergeführt werden. Wenn es gelingen würde, Teilaspekte der ESVP über Gemeinschaftsmittel zu finanzieren, dann wäre ein erster und wichtiger Schritt getan.

Eine einfache und kurzfristig realisierbare Lösung gibt es nicht: Die machtpolitisch sorgfältig austarierten Zuständigkeiten der Kommission, des Rates, der Nationen, des Europaparlamentes und der nationalen Parlamente führen dazu, dass Reformvorschläge oft vorschnell als Versuch abgetan werden, auf kaltem Wege die Machtverhältnisse zu verändern. Doch wer deshalb nichts tut, tut wohl auch das Falsche. Denn das Legitimationsdefizit der ESVP wächst weiter, je länger es andauert, obwohl es dringend überwunden werden muss. 


\subsection{Koalition der Parlamente: Der »Paritätische Ausschuss«}

Ein Weg, dieses Legitimationsdefizit zu reduzieren, besteht in einer »Allianz der Parlamente«. Vom Europäischen Parlament kann die Initiative für einen »Paritätischen Ausschuss « des Europaparlamentes und der nationalen Parlamente ausgehen, der unter Wahrung bestehender Zuständigkeiten und Rechte trotzdem schrittweise ein Mandat zur parlamentarischen Kontrolle der GASP und ESVP in allen ihren Aspekten, Initiativen und im Blick auf ihre weitere Ausgestaltung erhält.

In diesem Ausschuss kämen in einer ersten Phase gewählte Abgeordnete aus den nationalen Parlamenten und des Europaparlamentes zusammen, um sich gemeinsam über die ihnen jeweils zustehenden Auskünfte und Informationen auszutauschen und somit allen Beteiligten einen Gesamt- überblick von Initiativen im Bereich der GASP und der ESVP zu ermöglichen. Der gemeinsame Ausschuss könnte Vertreter der Exekutiven auf nationaler, intergouvernementaler und auf Kommissionsebene hören. Er sollte beratend den nationalen Parlamenten und Regierungen, aber auch der Kommission und dem Europäischen Rat über die Ergebnisse seiner Arbeit berichten und Empfehlungen unterbreiten. In einer zweiten Phase sollte die Arbeit des »Paritätischen Ausschusses « nach einigen Jahren auf eine rechtlich abgesicherte Basis gestellt werden, die es ihm ermöglicht, auch verbindliche Empfehlungen und Beschlüsse zu fassen.

Wird die Arbeit des Ausschusses für die Öffentlichkeit in hohem Maße transparent gestaltet, z.B. durch regelmäßige, öffentlich zugängliche Berichte über seine Arbeit und seine Empfehlungen, so kann er einen wesentlichen Beitrag zur Verringerung des drohenden Legitimations- und Transparenzdefizits der GASP, der ESVP und der künftigen EVP leisten.

\title{
Das europäische Sicherheitsforschungsprogramm Eine erste Einschätzung Martin Kahl/Achim Maas/Christian Mölling/Götz Neuneck*
}

\begin{abstract}
The European Security Strategy (ESS) not only furthered the common European security and defence policy, but also named for the first time a set of concrete threats for the security of the EU. The ESS furthermore formulated the objective to combine the efforts of the Union, thus enabling a common approach to counter these threats. One of the results is the European Security Research Program (ESRP) put forward by the European Commission. As we will show, the ESRP remains plagued by several inconsistencies which impair its effectiveness: while potentially providing viable solutions the program might also generate undesirable consequences due to these inherent problems. These potential risks and their management also mark the point of reference for a proposed interdisciplinary research program concerned with the impact of new security technologies on European societies and individuals.
\end{abstract}

Keywords: Europäische Kommission, Sicherheitsforschung, Technikfolgenabschätzung, Gefahrenabwehr, Terrorismus, Präventive Rüstungskontrolle

\section{Einführung}

A usgehend von den Bedrohungen, die in der European Security Strategy (ESS) ${ }^{1}$ aufgelistet werden, hat sich die EU-Kommission entschlossen, einen eigenen Beitrag zur inneren und äußeren Sicherheit der EU zu leisten. Zu diesem Zweck regte die Kommission das »European Security Research Programme« (ESRP) an, welches im 7. Forschungsrahmenprogramm (FP) ab 2007 jährlich mit voraussichtlich 1 Milliarde $€$ bezuschusst werden soll. Als Vorbereitung und

* Dr. Martin Kahl, Zentrum für Europäische Friedens- und Sicherheitsstudien (ZEUS) des Instituts für Friedensforschung und Sicherheitspolitik an der Universität Hamburg (IFSH); Achim Maas, Interdisziplinäre Forschungsgruppe Abrüstung und Rüstungskontrolle (IFAR) am IFSH; Christian Mölling, IFAR am IFSH; Dr. Götz Neuneck, Leiter IFAR am IFSH.

1 Solana, Javier: A Secure Europe in a Better World. European Security Strategy, angenommen durch den Rat der Europäischen Union am 12. Dezember 2003 in Brüssel. zur Entwicklung einer Forschungsagenda sollen 65 Mio. $€$ im Zeitraum von 2004 bis 2006 bereitgestellt werden. Eine zentrale Grundlage für dieses Vorgehen bildet der Bericht der »Group of Personalities« (GOP), welcher im März 2004 erschienen ist. ${ }^{2}$ Die GOP setzte sich neben hochrangigen Politikern vor allem aus Vertretern der (Rüstungs-)Industrie zusammen. Der Bericht bleibt in seiner Gefahrenanalyse wesentlich unbestimmter als die ESS, legt jedoch zugleich den Schwerpunkt auf technologische Innovationen, um den Bedrohungen zu begegnen. Die Grenzen zwischen zivilen und militärischen Anwendungsmöglichkeiten werden dabei nicht nur verwischt, die Entwicklung von Dual-Use-Technologien stellt vielmehr ein explizites Ziel des ESRP dar.

2 European Communities: Research for a Secure Europe. Report of the Group of Personalities in the Field of Security Research, Brüssel 2004. 